\title{
Morbilidade na Prematuridade Associada a Restrição do Crescimento Fetal e nos Prematuros Leves para a Idade Gestacional: Experiência de um Centro de Referência
}

\author{
Morbidity in Prematurity Associated with Fetal Growth \\ Restriction: Experience of a Tertiary Care Center
}

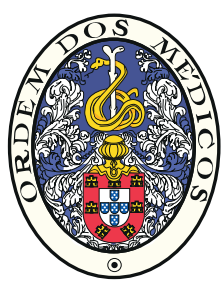

\author{
Noémia Rosado da SILVA $\bowtie 1$, Joana OLIVEIRA², Alberto BERENGUER ${ }^{3,4}$, André M. GRAÇA ${ }^{3,4}$, Margarida ABRANTES 3,4 , \\ Carlos MONIZ3,4 \\ Acta Med Port 2018 Nov;31(11):648-655 - https://doi.org/10.20344/amp.9599
}

\section{RESUMO}

Introdução: A prematuridade e o baixo peso ao nascer têm sido associados a maior morbilidade e mortalidade neonatais. Este estudo teve como objetivo avaliar possíveis fatores de risco para a prematuridade associada a restrição do crescimento fetal e a recém-nascidos leves para a idade gestacional e determinar a incidência da morbilidade nestes dois grupos de recém-nascidos.

Material e Métodos: Estudo caso-controlo retrospetivo dos recém-nascidos com idade gestacional inferior a 32 semanas, com o diagnóstico obstétrico de restrição do crescimento fetal e com o diagnóstico clínico de leves para a idade gestacional, internados na Unidade de Cuidados Intensivos Neonatais de um hospital terciário, durante um período de seis anos.

Resultados: Foram estudados 356 recém-nascidos, observando-se uma incidência de $11 \%$ de restrição do crescimento fetal e $18 \%$ de leves para a idade gestacional. A pré-eclâmpsia foi o fator de risco da gestação com maior significado estatístico ( $47 \%$ vs $16 \%, p<$ 0,001 ) nos recém-nascidos leves para a idade gestacional. Observou-se também, nestes recém-nascidos, maior incidência de displasia broncopulmonar ligeira ( $66 \%$ vs $38 \%, p=0,005)$, de sépsis tardia $(59 \%$ vs $37 \%, p=0,003)$, de retinopatia da prematuridade $(58 \%$ vs $26 \%, p=0,003)$ e de enterocolite necrotizante $(20 \%$ vs $9 \%, p=0,005)$. A mortalidade foi idêntica nos três grupos.

Discussão: Encontraram-se menos recém-nascidos do sexo masculino diagnosticados com restrição do crescimento fetal durante a gravidez comparativamente ao sexo feminino. Observaram-se diferenças significativas no grupo destes recém-nascidos, quanto à ocorrência de corioamnionite e de pré-eclâmpsia, face ao grupo controlo. Tanto os recém-nascidos com restrição do crescimento fetal como os leves para a idade gestacional apresentaram uma pontuação mais elevada nos índices de risco clínico comparativamente ao grupo controlo. De forma global, os recém-nascidos leves para a idade gestacional tiveram maior incidência de morbilidade que os recém-nascidos com restrição do crescimento fetal e que o grupo controlo.

Conclusão: Os avanços nos cuidados intensivos neonatais diminuíram a mortalidade nos recém-nascidos prematuros. Contudo, observam-se ainda diferenças significativas na incidência da morbilidade nos recém-nascidos com compromisso do crescimento. A colaboração entre obstetras e neonatalogistas constitui a base para uma correta avaliação clínica, sinalização precoce e intervenção global sobre estes recém-nascidos, com impacto significativo no prognóstico a curto e longo prazo.

Palavras-chave: Atraso do Crescimento Fetal; Desenvolvimento Fetal; Morbilidade; Recém-Nascido Pequeno para a Idade Gestacional; Recém-Nascido Prematuro

\section{ABSTRACT}

Introduction: Prematurity and low birth weight have been associated with increased neonatal morbidity and mortality. This study aimed to evaluate possible risk factors for prematurity associated with fetal growth restriction and being small for gestational age and to determine the incidence of morbidity in these two groups of infants.

Material and Methods: Retrospective case-control study of newborns with gestational age of less than 32 weeks, with obstetric diagnosis of fetal growth restriction and with the clinical diagnosis of small for gestational age, admitted to the Neonatal Intensive Care Unit of a tertiary hospital for a period of six years.

Results: A total of 356 newborns were studied, with an incidence of $11 \%$ of fetal growth restriction and $18 \%$ of small for gestational age. Pre-eclampsia was the risk factor for gestation with higher statistical significance $(47 \% v s 16 \%, p<0.001)$ in small for gestational age newborns. There was also a higher incidence of mild bronchopulmonary dysplasia $(66 \% v s 38 \%, p=0.005)$, late sepsis (59\% vs $37 \%, p=0.003)$, retinopathy of prematurity ( $58 \%$ vs $26 \%, p=0.003)$ and necrotizing enterocolitis $(20 \%$ vs $9 \%, p=0.005)$. Mortality was similar in all three groups.

Discussion: There were fewer newborn males diagnosed with fetal growth restriction during pregnancy compared to women. Significant differences were observed in the group of these infants regarding the occurrence of chorioamnionitis and pre-eclampsia in comparison to the control group. Newborns with fetal growth restriction and small for age had higher scores on clinical risk indices compared to the control group. In general, small for gestational age newborns had a higher incidence of morbidity than infants with fetal growth restriction and the control group.

Conclusion: Advances in neonatal intensive care decreased mortality in preterm infants. However, there are still significant differences in the incidence of morbidity in newborns with growth compromise. The collaboration between obstetricians and neonatologists provides the basis for a correct clinical evaluation, early signaling and global intervention on these newborns, with a significant impact on short and long-term prognosis.

1. Serviço de Pediatria. Unidade de Faro. Centro Hospitalar do Algarve. Faro. Portugal.

2. Serviço de Pediatria. Hospital de Santa Maria. Centro Hospitalar Lisboa Norte. Lisboa. Portugal.

3. Serviço de Neonatologia. Departamento de Pediatria. Hospital Universitário de Santa Maria. Centro Hospitalar Lisboa Norte. Lisboa. Portugal.

4. Clínica Universitária de Pediatria. Faculdade de Medicina. Universidade de Lisboa. Lisboa. Portugal.

$\triangle$ Autor correspondente: Noémia Rosado da Silva. noemialexandra@sapo.pt

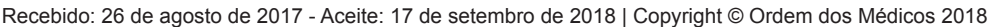


Keywords: Fetal Development; Fetal Growth Retardation; Infant, Premature; Infant, Small for Gestational Age; Morbidity

\section{INTRODUÇÃO}

Na última década, a indução do trabalho de parto e o número de cesarianas por indicação médica têm aumentado, com repercussão nas taxas da prematuridade. ${ }^{1}$

A prematuridade é uma das consequências da restrição do crescimento fetal (RCF) e a incidência de recém-nascidos (RN) leves para a idade gestacional (LIG) aumenta com a idade gestacional (IG) mais baixa. ${ }^{2} \mathrm{O}$ parto prematuro e a restrição do crescimento representam um risco acrescido para o RN porque cada uma destas condições está associada a complicações neonatais. ${ }^{3}$

A RCF refere-se à condição na qual o feto não atinge o seu potencial genético de crescimento, ${ }^{4,5}$ embora não exista um consenso internacional para a sua definição. ${ }^{6}$ Este diagnóstico é dado pelo registo da velocidade de crescimento fetal, obtido através de avaliações obstétricas seriadas $^{7}$ e estima-se que a sua prevalência afete $5 \%$ a $7 \%$ das gestações. ${ }^{8}$ A causa major desta patologia é a insuficiência placentária. ${ }^{8}$ Por outro lado, o diagnóstico de RN LIG é clínico e define-se como o RN com crescimento abaixo do percentil 10 para a sua IG. Contudo, após o nascimento, o peso inferior ao percentil 10 para a IG é amplamente utilizado como medida de restrição do crescimento. ${ }^{9}$ Deste modo, as vantagens e desvantagens das duas medidas de crescimento, bem como a seleção de padrões e percentis são uma questão de debate contínuo. ${ }^{9}$ Sabe-se que nem todos os RN LIG apresentam RCF, podendo ser somente constitucionalmente pequenos. ${ }^{10}$ Da mesma forma, nem todos os fetos que não atingiram o seu potencial de crescimento genético se encontram abaixo do percentil 10 para o peso esperado. ${ }^{2,4}$ As causas podem ter origem materna, placentária ou fetal e a gravidade da RCF e o prognóstico do RN LIG dependem da etiologia. ${ }^{11}$ Alguns autores referem ainda tendências seculares no peso ao nascer mediadas por fatores intergeracionais. ${ }^{12}$

Estão descritos como fatores de risco maternos associados à RCF, a idade materna avançada, excesso de peso ou baixo peso pré-gestação, hábitos toxicofílicos, profissão, nível socioeconómico e reprodução medicamente assistida. ${ }^{6,13-15}$ Outras condições estabelecidas incluem pré-eclâmpsia, hipertensão induzida pela gravidez, doença autoimune, diabetes e hipertensão essencial. ${ }^{13,16}$ Autores afirmam que a prevalência de LIG é um bom indicador da saúde e estado nutricional maternos. ${ }^{17}$

Quanto aos fatores placentários descreve-se a alteração do tamanho e função da placenta com consequente disfunção da troca de nutrientes entre mãe e feto, fatores endócrinos, de crescimento e citoquinas. ${ }^{18}$ Dentro das causas fetais, identificam-se as malformações, as infeções congénitas do grupo TORCH (toxoplasmose, rubéola, sífilis, citomegalovírus e herpes vírus) e as alterações genéticas. ${ }^{11}$

Juntamente com a prematuridade, a condição de RCF e/ou LIG tem sido associada a maior morbilidade e mortalidade durante o primeiro ano de $v^{1 d a^{8,19}} \mathrm{e}$ a risco aumentado para diversas patologias na idade adulta..$^{1,20}$ No período neonatal observa-se maior incidência de asfixia, hipotermia, acidose metabólica, policitemia, hipoglicémia e hipocalcémia. ${ }^{11}$ No primeiro ano de vida observam-se alterações do desenvolvimento psicomotor ${ }^{8,15}$ e deficiente recuperação somatométrica. ${ }^{21} \mathrm{Na}$ idade adulta há evidências de maior predisposição para sofrer síndrome metabólico, acidente vascular cerebral e recorrência de baixo peso ao nascer na descendência. ${ }^{1,22-24}$

Embora os RN prematuros constituam apenas 5\% $10 \%$ dos nascimentos, representam mais de dois terços das mortes neonatais $(35 \%-60 \%$ se IG inferior a 32 semanas), sendo um importante desafio para os sistemas de saúde, pela necessidade de cuidados médico-cirúrgicos altamente especializados..$^{19}$

O objetivo deste estudo foi a determinação da incidência de RN prematuros com diagnóstico pré-natal de RCF e de RN prematuros LIG na nossa unidade a avaliação de possíveis fatores de risco da gestação/parto e a caracterização da morbilidade neonatal associada a estas situações, nomeadamente quanto à incidência de persistência do canal arterial, enterocolite necrotizante, sépsis tardia, retinopatia da prematuridade, complicações neurológicas e displasia broncopulmonar.

\section{MATERIAL E MÉTODOS \\ Tipo de estudo}

Estudo caso-controlo desenvolvido através da recolha retrospetiva de dados da base nacional Registo Nacional de RN de Muito Baixo Peso.

\section{Participantes}

Foram incluídos $\mathrm{RN}$ com diagnóstico de prematuridade, RCF e LIG internados na Unidade de Cuidados Intensivos Neonatais (UCIN) do Hospital de Santa Maria, no período de seis anos, compreendido entre 1 de janeiro de 2010 e 31 dezembro de 2015.

Os critérios de inclusão foram IG até às 31 semanas e seis dias de gestação, diagnóstico obstétrico de RCF (peso inferior ao percentil 10 e alteração na fluxometria doppler da artéria umbilical) ${ }^{25}$ e/ou diagnóstico clínico de LIG (peso inferior ao percentil 10 para a IG de acordo com os gráficos de Fenton de $2013,{ }^{26}$ com distinção entre géneros).

\section{Recolha de dados}

Os dados foram colhidos através da análise dos dados relativos à nossa unidade constante do Registo Nacional de RN de Muito Baixo Peso.

Foi avaliada a presença dos seguintes fatores de risco durante a gestação: pré-eclâmpsia, diabetes, corioamnionite e hemorragia precoce. Foram também consideradas as seguintes características da gestação: espontânea ou por fertilização in vitro (FIV), simples ou gemelar; e o tipo de parto: vaginal ou por cesariana. 
Relativamente ao RN, os parâmetros avaliados foram: IG, sexo, peso ao nascimento (PN), indução maturativa pulmonar fetal, índice de Apgar, CRIB (clinical risk index for babies), SNAP-PE (score for neonatal acute physiology - perinatal extension), necessidade de intubação endotraqueal (EET), necessidade de ventilação invasiva (VI) ou não invasiva (VNI), persistência do canal arterial (PCA) com indicação de tratamento médico ou cirúrgico, sépsis tardia, enterocolite necrotizante, necessidade de oxigénio suplementar aos 28 dias e às 36 semanas, ${ }^{27}$ retinopatia da prematuridade (ROP), duração do internamento e alterações na ecografia transfontanelar: leucomalácia periventricular (LPV), hemorragia peri-intraventricular (HPIV) e enfarte hemorrágico periventricular (EHPV). Foram também avaliadas as complicações médicas: hidrocefalia com necessidade de derivação ventrículo-peritoneal (DVP) e necessidade de oxigénio suplementar à data da alta. Foram excluídos do estudo RN com malformações congénitas.

\section{Análise de dados}

As amostras (grupo constituído por RN com o diagnóstico pré-natal de RCF e grupo constituído por RN LIG) foram comparadas com um grupo controlo constituído pelos RN com a mesma IG, mas com somatometria adequada à idade gestacional e sem diagnóstico obstétrico de RCF, internados na mesma UCIN, no período do estudo.

A análise dos dados foi realizada utilizando os programas informáticos Microsoft Exce ${ }^{\circledR} 2013$ e SPSS ${ }^{\circledR} 20$. Foi usado o teste de Mann-Whitney para comparação de médias; o teste de qui-quadrado e o teste exato de Fisher para comparação de variáveis dicotómicas; nas variáveis IG e PN foram utilizadas a mediana como medida de localização central e o intervalo interquartil como medida de dispersão. Considerou-se um nível de significância de $5 \%$.

\section{RESULTADOS}

Foram contabilizados no total 356 RN com IG inferior a 32 semanas (mediana da IG de 29 semanas) internados na UCIN no período de 2010 a 2015. Destes, 40 RN tinham diagnóstico de RCF, correspondendo a uma incidência de $11 \%$, e 63 eram LIG (51 com PN inferior ao P10 e $12 \mathrm{com}$ PN inferior ao P3), correspondendo a uma incidência de $18 \%$. A mediana do PN foi de $900 \mathrm{~g}$ nos RN com RCF, 1150 g nos RN LIG e 1155 g no grupo controlo. Apenas 22 RN LIG foram diagnosticados como RCF.

A distribuição dos óbitos por idade gestacional e peso ao nascimento no grupo de RN RCF/LIG e no grupo controlo apresenta-se na Tabela 1.

Observou-se um discreto predomínio no sexo feminino no que diz respeito aos diagnósticos de RCF $(52 \%)$ e de LIG (57\%), comparativamente ao grupo controlo (44\%).

Apresentaram patologia durante a gravidez $44 \%$ das mães de RN com RCF e $67 \%$ das mães de RN LIG, em contraste com $51 \%$ no grupo controlo. Dentro da patologia da gestação, a pré-eclâmpsia foi documentada em $47 \%$ das mães dos RN LIG, face a $16 \%$ no grupo controlo,

Tabela 1 - Distribuição dos óbitos por idade gestacional e peso ao nascimento no grupo de RN RCF/LIG e no grupo controlo

\begin{tabular}{|c|c|c|c|c|c|c|}
\hline IG (semanas) & RCF/LIG (n) & PN (g) Mín-Máx & Óbitos (n) & Controlo (n) & PN (g) Mín - Máx & Óbitos (n) \\
\hline 23 & 0 & NA & NA & 2 & $545-550$ & 2 \\
\hline 24 & 4 & $470-500$ & 3 & 9 & $510-799$ & 8 \\
\hline 25 & 4 & $430-560$ & 3 & 16 & $600-930$ & 6 \\
\hline 26 & 14 & $530-649$ & 1 & 22 & $639-1100$ & 4 \\
\hline 27 & 12 & $300-680$ & 2 & 28 & $720-1230$ & 2 \\
\hline 28 & 25 & $549-810$ & 2 & 43 & $750-1409$ & 5 \\
\hline 29 & 7 & $568-924$ & 0 & 48 & $950-1640$ & 4 \\
\hline 30 & 21 & $640-1043$ & 0 & 47 & $930-1790$ & 2 \\
\hline 31 & 16 & $909-1200$ & 0 & 60 & $1110-2240$ & 2 \\
\hline Total & 103 & & $11(11 \%)$ & 275 & & $35(13 \%)$ \\
\hline
\end{tabular}

IG: idade gestacional; RCF: restrição do crescimento fetal; LIG: leve para a idade gestacional; PN: peso ao nascimento; Mín: mínimo; Máx:máximo; NA: não aplicável Tabela 2 - Fatores de risco da gestação e parto - comparação entre RN LIG e grupo controlo

\begin{tabular}{|c|c|c|c|c|c|}
\hline Variáveis & LIG (n) & $\%$ & Controlo (n) & $\%$ & $p$ \\
\hline Idade gestacional (semanas), média (mín - máx) & \multicolumn{2}{|c|}{27,9} & \multicolumn{2}{|c|}{28,6} & $<0,01$ \\
\hline Espontânea & 47 & 75 & 213 & 77 & NS \\
\hline FIV & 16 & 25 & 62 & 23 & NS \\
\hline Cesariana & 37 & 93 & 204 & 65 & $<0,001$ \\
\hline Gemelaridade & 20 & 31 & 109 & 39 & NS \\
\hline Pré-eclampsia & 30 & 47 & 45 & 16 & $<0,001$ \\
\hline Diabetes & 3 & 5 & 26 & 9 & NS \\
\hline Hemorragia precoce & 8 & 12 & 29 & 10 & NS \\
\hline Corioamnionite & 2 & 3 & 46 & 16 & NS \\
\hline
\end{tabular}

LIG: leve para a idade gestacional; FIV: fertilização in vitro; NS: não significativo 
Tabela 3 - Fatores de risco da gestação e parto - comparação entre RCF e grupo controlo

\begin{tabular}{|c|c|c|c|c|c|}
\hline Variáveis & $\operatorname{RCF}(n)$ & $\%$ & Controlo (n) & $\%$ & $p$ \\
\hline Idade gestacional (semanas), média (mín - máx) & \multicolumn{2}{|c|}{28,9} & \multicolumn{2}{|c|}{28,4} & NS \\
\hline Espontânea & 20 & 50 & 213 & 77 & NS \\
\hline FIV & 20 & 50 & 62 & 23 & NS \\
\hline Cesariana & 53 & 84 & 188 & 64 & $<0,005$ \\
\hline Gemelaridade & 13 & 32 & 109 & 39 & NS \\
\hline Pré-eclampsia & 13 & 32 & 45 & 16 & NS \\
\hline Diabetes & 2 & 5 & 26 & 9 & NS \\
\hline Hemorragia precoce & 2 & 5 & 29 & 10 & NS \\
\hline Corioamnionite & 1 & 2 & 46 & 16 & $<0,005$ \\
\hline
\end{tabular}

RCF: restrição do crescimento fetal, FIV: fertilização in vitro; NS: não significativo

correspondendo a uma diferença significativa $(p<0,001)$. Verificou-se gemelaridade em cerca de $30 \%$ dos RN com RCF e RN LIG. A maioria das gestações foi espontânea no caso dos RN LIG (75\%), face a apenas metade (50\%) das gestações dos RN com RCF. Observou-se maior número de partos por cesariana, superior a $80 \%$, em ambos grupos. Estes números não apresentam diferença significativa comparativamente ao grupo controlo. Os valores referentes à comparação da IG, patologia durante a gestação e ao tipo de parto apresentam-se detalhadamente nas Tabelas 1, 2 e 3.
Quanto à morbilidade nos grupos em estudo, necessitaram de VI $60 \%$ dos RN LIG e $50 \%$ dos RN com RCF em comparação com $47 \%$ no grupo controlo, e de VNI $84 \%$ dos RN LIG e $87 \%$ dos RN com RCF em comparação com $53 \%$ no grupo controlo. Apresentaram necessidade de oxigénio suplementar aos 28 dias, $66 \%$ dos RN LIG e 55\% das RCF, face a $38 \%$ no grupo controlo (diferença significativa no grupo LIG, $(p=0,005)$. Outras variáveis com diferenças significativas nos RN LIG foram: sépsis tardia (59\% vs $37 \%, p=0,003)$, ROP (58\% vs $26 \%, p=0,003$ ) e enterocolite necrotizante ( $20 \%$ vs $9 \%, p=0,005)$. Nos RN com

Tabela 4 - Morbilidade Neonatal - comparação entre LIG e grupo controlo.

\begin{tabular}{|c|c|c|c|c|c|}
\hline Variável & LIG (n) & $\%$ & Controlo (n) & $\%$ & $p$ \\
\hline IMPF completa & 44 & 70 & 191 & 69 & $\mathrm{NS}^{\mathrm{a}}$ \\
\hline Duração do internamento (média em dias) & 86 & NA & 53 & NA & $<0,001^{b}$ \\
\hline Índice de Apgar 1 min (média de pontuação) & 7 & NA & 7 & NA & NA \\
\hline Índice de Apgar 5 min (média de pontuação) & 9 & NA & 9 & NA & NA \\
\hline Reanimação com intubação & 44 & 70 & 174 & 63 & NS ${ }^{a}$ \\
\hline CRIB (média de pontuação) & 5,3 & NA & 2,5 & NA & $<0,001^{b}$ \\
\hline SNAP-PE (média de pontuação) & 31,7 & NA & 17,2 & NA & $<0,01^{b}$ \\
\hline Ventilação invasiva & 38 & 60 & 129 & 47 & $\mathrm{NS}^{\mathrm{a}}$ \\
\hline Ventilação não invasiva & 53 & 84 & 146 & 53 & $\mathrm{NS}^{\mathrm{a}}$ \\
\hline Enterocolite necrotizante & 13 & 20 & 24 & 9 & $<0,01^{\mathrm{a}}$ \\
\hline PCA tratamento médico & 17 & 27 & 52 & 19 & $\mathrm{NS}^{\mathrm{a}}$ \\
\hline PCA tratamento cirúrgico & 6 & 9 & 12 & 4 & $\mathrm{NS}^{\mathrm{a}}$ \\
\hline Oxigénio aos 28 dias & 34 & 66 & 105 & 38 & $<0,01^{\mathrm{a}}$ \\
\hline Oxigénio às 36 semanas & 17 & 34 & 41 & 15 & $\mathrm{NS}^{\mathrm{a}}$ \\
\hline Sépsis Tardia & 37 & 59 & 103 & 37 & $<0,005^{2}$ \\
\hline ROP & 27 & 58 & 73 & 26 & $<0,005^{2}$ \\
\hline Hemorragia peri-intraventricular & 10 & 16 & 63 & 23 & $N^{a}$ \\
\hline Enfarte venoso hemorrágico periventricular & 3 & 5 & 9 & 3 & $\mathrm{NS}^{\mathrm{c}}$ \\
\hline Leucomalácia periventricular quística & 4 & 9 & 22 & 8 & $\mathrm{NS}^{\mathrm{c}}$ \\
\hline Complicações & LIG (n) & $\%$ & Controlo (n) & $\%$ & $p$ \\
\hline Hidrocefalia com DVP & 1 & 4 & 14 & 5 & $\mathrm{NS}^{\mathrm{c}}$ \\
\hline Oxigénio à data da alta & 7 & 27 & 16 & 6 & $\mathrm{NS}^{\mathrm{c}}$ \\
\hline
\end{tabular}

LIG: leve para a idade gestacional; IMPF: indução maturativa pulmonar fetal; CRIB: clinical risk index for babies; SNAP-PE: score for neonatal acute physiology perinatal extension; PCA: Ppersistência do canal arterial; ROP: retinopatia da prematuridade; DVP: derivação ventrículo-peritoneal; NS: não significativo; NA: não aplicável; a teste de qui-quadrado, b teste de Mann-Whitney-U, ${ }^{\circ}$ teste exato de Fisher, $p<0,05$. 
RCF a sépsis tardia foi a única patologia com significância estatística $(p=0,04)$.

A principal complicação documentada foi a necessidade de oxigénio suplementar à data da alta nos RN LIG (27\%). Os dados referentes à morbilidade apresentam-se nas Tabelas 4 e 5 .

\section{DISCUSSÃO}

Neste estudo pretendeu-se avaliar possíveis fatores de risco da gestação/parto para a prematuridade associada a RCF e/ou RN LIG e determinar a incidência da morbilidade e mortalidade nestes RN. A estimativa do peso fetal e a deteção pré-natal de RCF desempenham um papel significativo nas decisões do obstetra, quanto às indicações para indução do parto e no aconselhamento parental. Em contrapartida, o percentil de crescimento do RN, calculado pelo peso ao nascimento, é a informação chave usada pelos pediatras na tomada de decisões na unidade neonatal. ${ }^{9}$ Na nossa amostra, a incidência no RN prematuro de RCF foi de $11 \%$ e de LIG foi de $18 \%$, provavelmente pelo facto do hospital ser um centro de referência nacional obstétrico e neonatal. No nosso estudo, dois terços dos RN LIG não foram diagnosticados como RCF durante a gravidez. Os estudos referem que, embora a ecografia seja o exame gold standard para deteção de RCF, o erro associado à estimativa de peso é de aproximadamente $20 \% .{ }^{28,29}$ Defende- -se ainda, que uma das razões para este resultado, possa ser o uso de curvas de crescimento na prática clínica, que não estão adaptadas aos grandes prematuros. ${ }^{30,31}$

\section{Fatores de risco da gestação e parto}

Zeitlin et al descrevem uma forte associação entre o peso ao nascimento e complicações da gravidez associadas ao parto prematuro. ${ }^{32} \mathrm{Na}$ análise retrospetiva da gestação e parto, o nosso estudo avaliou alguns dos fatores de risco provavelmente responsáveis pelo baixo peso ao nascer, abordados na literatura. ${ }^{6} \mathrm{Na}$ nossa amostra, não se constaram diferenças entre os grupos estudados em relação ao tipo de gestação (espontânea ou por FIV). De igual modo, um terço dos RN com RCF e RN LIG resultaram de uma gestação gemelar, à semelhança do grupo controlo, pelo que esta variável não constituiu um fator significativo. Quanto à patologia durante a gravidez, observaram-se diferenças significativas no grupo dos RN com RCF, quanto à ocorrência de corioamnionite. Foi também encontrada uma elevada percentagem de casos de pré-eclâmpsia, face ao grupo controlo, nos RN LIG (47\% vs 16\%, $p<0,001)$, constituindo sem dúvida, a patologia da gestação mais fortemente associada a morbilidade neonatal.

\section{Diferença entre géneros}

À semelhança da nossa amostra, Monier et al e

Tabela 5 - Morbilidade neonatal - comparação entre RCF e grupo controlo

\begin{tabular}{|c|c|c|c|c|c|}
\hline Variável & RCF (n) & $\%$ & Controlo (n) & $\%$ & $p$ \\
\hline IMPF completa & 36 & 90 & 191 & 69 & $<0,01^{\mathrm{a}}$ \\
\hline Duração do internamento (média em dias) & 72 & NA & 53 & NA & $N S^{b}$ \\
\hline Índice de Apagar 1 min (média de pontuação) & 8 & NA & 7 & NA & NA \\
\hline Índice de Apgar 5 min (média de pontuação) & 10 & NA & 9 & NA & NA \\
\hline Reanimação com intubação & 21 & 52 & 174 & 63 & $\mathrm{NS}^{\mathrm{a}}$ \\
\hline CRIB (média de pontuação) & 3,9 & NA & 2,5 & NA & $<0,01$ \\
\hline SNAP-PE (média de pontuação) & 21,8 & NA & 17,2 & NA & $<0,05$ \\
\hline Ventilação invasiva & 20 & 50 & 129 & 47 & NS ${ }^{a}$ \\
\hline Ventilação não invasiva & 35 & 87 & 146 & 53 & NS ${ }^{a}$ \\
\hline Enterocolite necrotizante & 5 & 12 & 24 & 9 & NS ${ }^{a}$ \\
\hline PCA tratamento médico & 8 & 20 & 52 & 19 & $\mathrm{NS}^{\mathrm{a}}$ \\
\hline PCA tratamento cirúrgico & 3 & 7 & 12 & 4 & $\mathrm{NS}^{\mathrm{c}}$ \\
\hline Oxigénio aos 28 dias & 22 & 55 & 105 & 38 & $\mathrm{NS}^{\mathrm{a}}$ \\
\hline Oxigénio às 36 semanas & 8 & 20 & 41 & 15 & NS ${ }^{a}$ \\
\hline Sépsis Tardia & 23 & 57 & 103 & 37 & $<0,05^{\mathrm{a}}$ \\
\hline ROP & 15 & 37 & 73 & 26 & $\mathrm{NS}^{\mathrm{a}}$ \\
\hline Hemorragia peri-intraventricular & 5 & 12 & 63 & 23 & NS ${ }^{a}$ \\
\hline Enfarte venoso hemorrágico periventricular & 4 & 10 & 9 & 3 & $\mathrm{NS}^{\mathrm{c}}$ \\
\hline Leucomalácia periventricular quística & 3 & 7 & 22 & 8 & $\mathrm{NS}^{\mathrm{c}}$ \\
\hline Complicações & $\operatorname{RCF}(n)$ & $\%$ & Controlo (n) & $\%$ & $p$ \\
\hline Hidrocefalia com DVP & 1 & 16 & 14 & 5 & $\mathrm{NS}^{\mathrm{c}}$ \\
\hline Oxigénio à data da alta & 0 & 0 & 16 & 6 & $\mathrm{NS}^{\mathrm{c}}$ \\
\hline
\end{tabular}

RCF: restrição do crescimento fetal; IMPF: indução maturativa pulmonar fetal; CRIB: clinical risk index for babies; SNAP-PE: score for neonatal acute physiology perinatal extension; PCA: persistência do canal arterial; ROP: retinopatia da prematuridade; DVP: derivação ventrículo-peritoneal; NS: não significativo; NA: não aplicável; a teste de qui-quadrado, ${ }^{b}$ teste de Mann-Whitney-U, ${ }^{c}$ teste exato de Fisher, $p<0,05$. 
Magalhães et al encontraram menos RN do sexo masculino diagnosticados como RCF durante a gravidez comparativamente ao sexo feminino, provavelmente explicado pelo uso de referências de crescimento não ajustadas em função do sexo do RN, podendo também adaptar-se ao caso dos RN LIG. $^{30,33}$

Sublinhamos que a inexistência de um padrão descritivo da somatometria ao nascimento da população portuguesa torna necessário o uso de padrões gráficos internacionais, com consequente desajuste entre estes e as caraterísticas de cada população. Deve ser sublinhado que a população do nosso hospital apresenta uma grande heterogeneidade sociocultural, recebendo tanto imigrantes europeus, como oriundos do continente africano e asiático. Este desajuste na classificação dos RN tem ainda repercussões na determinação do prognóstico individual e nas consequentes intervenções médicas que este pode condicionar. ${ }^{34}$

Cabe realçar, que a limitação do nosso estudo foi o facto de não ter sido determinada a estatura dos progenitores dos RN LIG.

\section{Avaliação dos scores CRIB e SNAP-PE}

O CRIB (clinical risk index for babies) avalia a gravidade clínica inicial em recém-nascidos prematuros com base no peso de nascimento, idade gestacional, existência de malformação congénita, valor de excesso de bases e de fração inspirada de oxigênio. Foi desenvolvido pelo International Neonatal Network no Reino Unido em 1993 e é útil para medir o risco de óbito hospitalar. ${ }^{35}$ Os nossos resultados mostraram uma média de pontuação máxima de 5,3 valores nos RN LIG $(p<0,001)$ e de 3,9 valores nos RN com RCF $(p<0,01)$. No grupo controlo, o grau de gravidade do score foi notavelmente inferior, com uma média de pontuação de 2,5. Apesar de, na prática clínica, os três grupos corresponderem a um CRIB grau I, as diferenças são significativas. Os resultados obtidos evidenciam uma tendência para um maior risco clínico nos grupos estudados, especialmente nos RN LIG.

O SNAP-PE (score for neonatal acute physiology - perinatal extension) é uma extensão da escala SNAP (score for neonatal acute physiology) desenvolvido por Richardson et a ${ }^{\beta 6}$ para avaliar a gravidade clínica dos RN na admissão numa UCIN. Considera todos os parâmetros fisiológicos do SNAP e avalia também o peso de nascimento, os dados da história perinatal, como o índice de Apgar, e a classificação de RN LIG. ${ }^{37,38}$ Assim como observado nos resultados da aplicação do CRIB, os RN com RCF/LIG apresentaram uma pontuação mais elevada no SNAP-PE comparativamente ao grupo controlo (31,7 nos LIG e 21,8 nos RCF vs 17,2 no grupo controlo). Novamente, a diferença de pontuação nesta escala de classificação foi significativa em ambos grupos, confirmando-se o maior risco de pior prognóstico nestes RN.

\section{Displasia broncopulmonar}

Um estudo recente observou um aumento de displasia broncopulmonar (DBP) nos RN LIG, independentemente de apresentarem diagnóstico pré-natal de RCF. ${ }^{30}$ Noutra publicação, o risco de DBP foi também fortemente associado aos RN LIG, observando-se a sua diminuição com o aumento do peso ao nascimento em todo o espectro. ${ }^{32}$ No nosso caso, a necessidade de oxigénio suplementar aos 28 dias de vida alcançou $66 \%$ nos LIG $(p=0,005)$ e às 36 semanas revelou-se o dobro da encontrada no grupo controlo ( $34 \%$ vs 15\%), embora esta última não significativa. Também no grupo dos RN LIG, a complicação principal foi a dependência de oxigénio à data da alta (27\%), não observada nas RCF no nosso estudo. Este facto corrobora os achados da literatura, mas poderá também dever-se à realização de maturação pulmonar fetal completa em apenas $70 \%$ dos RN LIG, comparativamente a $90 \%$ dos RN com RCF.

\section{Implicações neurológicas}

Os RN extremamente prematuros exibem alterações no volume cerebral, sensíveis ao risco perinatal múltiplo, aos fatores de morbilidade e intervenções neonatais. ${ }^{39,40}$ Vários estudos, incluindo um estudo realizado pelo nosso grupo, ${ }^{40}$ demonstraram uma associação entre volumes cerebrais regionais mais pequenos e o comprometimento neurológico nos prematuros. ${ }^{39-41}$ Estas reduções de volume acentuadas foram acompanhadas pelo aumento do líquido cefalorraquidiano (LCR) em detrimento de um menor tamanho global do crânio, indicando atrofia cerebral. ${ }^{39,40}$

Num outro estudo verificou-se que os gânglios da base e o tálamo são significativamente menores nos RN com RCF em comparação com os RN com peso adequado à idade gestacional. ${ }^{42}$ Estas alterações poderão ter implicações clínicas quanto à aprendizagem e memória, na função linguística e na função visual-espacial. ${ }^{42}$

A hemorragia peri-intraventricular (HPIV > 2) no prematuro é uma lesão adquirida com um impacto direto na morbilidade, mortalidade e evolução do neurodesenvolvimento a longo prazo. ${ }^{43}$ No entanto, no estudo de Zeitlin et al, a condição de LIG não foi associada a maior incidência de HPIV ou de LPV quística. ${ }^{32}$ No nosso estudo, a percentagem de HPIV nos RN LIG atingiu $16 \%$ e nos RN com RCF $12 \%$. A ocorrência de LPV quística foi de $9 \%$ nos RN LIG e $7 \%$ nos com RCF, não se verificando diferenças significativas face ao grupo controlo.

\section{Mortalidade e morbilidade}

Os Modelos para a Organização do Acesso ao Cuidado Intensivo para Recém-nascidos com Prematuridade Extrema na Europa (MOSAIC), ${ }^{44}$ um estudo coorte populacional de crianças nascidas antes das 32 semanas de gestação, relatou maior risco de mortalidade e morbilidade associada a um peso ao nascimento inferior ao percentil 10. Contudo, estudos demonstram que a deteção e orientação precoces dos fetos com RCF diminuem significativamente (cerca de $20 \%$ ) a morbilidade e mortalidade perinatal. ${ }^{45}$ Porventura uma abordagem obstétrica e perinatal adequada explicam a ausência de diferença significativa na mortalidade entre os RN com RCF e o grupo de controlo.

$\mathrm{Na}$ nossa amostra, quanto à análise da incidência de 
morbilidade, constatou-se maior percentagem tanto nas RCF $(p<0,05)$ como nos RN LIG $(p<0,005)$ de sépsis tardia, comparativamente ao grupo controlo. A percentagem de sépsis tardia nestes RN poderá ser explicada pelo início mais tardio da alimentação entérica, que tem como consequência o aumento da duração da alimentação parentérica e da necessidade de cateterismo central. RN LIG apresentaram maior incidência de ROP (58\% vs $26 \%$, $p<0,005)$ e de enterocolite necrotizante, face ao grupo controlo $(20 \%$ vs $9 \%, p<0,01$ ), com diferenças significativas.

De forma global, no nosso estudo, os RN LIG apresentaram maior incidência de morbilidade que os RN com RCF e que o grupo controlo. Estes resultados parecem apoiar a hipótese de que o baixo percentil ponderal ao nascimento traduz o défice real existente a nível do desenvolvimento orgânico.

\section{Limitações do estudo}

1. Houve menos RN do sexo masculino diagnosticados como RCF durante a gravidez comparativamente ao sexo feminino, provavelmente explicado pelo uso de referências de crescimento não ajustadas em função do sexo do RN, podendo também adaptar-se ao caso dos RN LIG.

2. A população do nosso hospital apresenta uma grande heterogeneidade sociocultural, podendo ocorrer um desajuste na classificação dos RN que venha a provocar repercussões na determinação do prognóstico individual e nas consequentes intervenções médicas que este pode condicionar.

3. Não foi determinada a estatura dos progenitores dos RN LIG.

Pontos fortes:

1. Este estudo foi desenvolvido com base na experiência de um centro de referência em Cuidados Intensivos Neonatais, recorrendo a uma amostra de 356 RN com IG inferior a 32 semanas.

2. Foram analisados dezassete parâmetros clínicos para avaliação da morbilidade destes RN, assim como as características da gestação e os principais fatores de risco durante a mesma, descritos na literatura.

O que é que este estudo acrescenta à literatura nacional?

Este estudo reavaliou os possíveis fatores de risco da gestação/parto para a prematuridade associada a RCF e/ ou RN LIG, observando que a pré-eclâmpsia constituiu a patologia da gestação mais fortemente associada a morbilidade neonatal.

Neste estudo constatou-se ainda que os RN LIG apresentam maior incidência de morbilidade que os RN com RCF e que o grupo controlo, apoiando a hipótese de que o baixo percentil ponderal ao nascimento traduz o défice real existente a nível do desenvolvimento orgânico.

\section{CONCLUSÃO}

Os avanços nos cuidados intensivos neonatais aumentaram significativamente a sobrevida e diminuíram a morbilidade e a mortalidade nos RN prematuros. Contudo, observam-se ainda diferenças significativas quanto à prevalência de DBP, enterocolite necrotizante, sépsis tardia e retinopatia da prematuridade nos RN LIG e de sépsis tardia nos RN com RCF. O nosso estudo demonstrou também uma forte associação entre RN LIG e pré-eclâmpsia materna.

O compromisso do crescimento fetal, resultante num baixo peso ao nascer para a IG e sexo, mostrou ter maior implicação na morbilidade do RN, comparativamente aos casos com diagnóstico pré-natal de RCF sem compromisso de crescimento.

A comunicação e colaboração ativa entre obstetras e neonatalogistas constituem assim, a base para uma correta avaliação clínica, sinalização e intervenção global sobre estes RN, minimizando a morbilidade precoce e as repercussões tardias.

\section{PROTECÇÃO DE PESSOAS E ANIMAIS}

Os autores declaram que os procedimentos seguidos estavam de acordo com os regulamentos estabelecidos pelos responsáveis da Comissão de Investigação Clínica e Ética e de acordo com a Declaração de Helsínquia da Associação Médica Mundial.

\section{CONFIDENCIALIDADE DOS DADOS}

Os autores declaram ter seguido os protocolos do seu centro de trabalho acerca da publicação de dados.

\section{CONFLITOS DE INTERESSE}

Os autores declaram não terem qualquer conflito de interesse relativamente ao presente artigo.

\section{FONTES DE FINANCIAMENTO}

Os autores declaram não ter recebido subsídios ou bolsas para a elaboração do artigo.

\section{REFERÊNCIAS}

1. Coutinho P, Cecatti J, Surita F, Costa M, Morais S. Perinatal outcomes associated with low birthweight in a historical cohort. Reprod Health. 2011;8:1-6.

2. Alexandrino A. Período neonatal - restrição de crescimento intrauterino: decisões obstétricas com repercussão no $\mathrm{RN}$ - mesa redonda, XXIV Reunião Anual de Pediatria do Centro Hospitalar do Porto. Nascer Crescer. 2012;21:167-8.

3. Doyle L, Victorian Infant Collaborative Study Group. Outcome at 5 years of age of children 23 to 27 weeks' gestation: refining the prognosis.
Pediatrics. 2001;108:134-41.

4. Ross M. Fetal growth restriction: overview, causes of intrauterine growth restriction, perinatal implications. New York: Medscape; 2015.

5. Grandi C, Tapia J, Marshall G, Grupo Colaborativo NEOCOSUR Evaluación de la severidad, proporcionalidad y riesgo de muerte de recién nacidos de muy bajo peso con restricción del crecimiento fetal. Análisis multicéntrico sudamericano. J Pediatr. 2005;81:198-204.

6. Gaudineau A. Prévalence, facteurs de risque et morbi-mortalité materno-foetale des troubles de la croissance foetale. J Gynecol Obstet 
Biol Reprod. 2013;42:895-910.

7. Magalhães J, Resende C, Braga A, Alexandrino A. Muito baixo peso e restrição de crescimento intrauterino - uma associação de mau prognóstico? Acta Pediatr Port. 2014;45:107-15.

8. Levine T, Grunau R, McAuliffe F, Pinnamaneni R, Foran A, Alderdice F. Early childhood neurodevelopment after intrauterine growth restriction: a systematic review. Pediatrics. 2015;135:126-41.

9. El Ayoubi M, Jarreau P, Van Reempts P, Cuttini M, Kaminski M, Zeitlin J et al. Does the antenatal detection of fetal growth restriction (FGR) have a prognostic value for mortality and short-term morbidity for very preterm infants? Results from the MOSAIC cohort. J Matern Fetal Neonatal Med. 2016;29:596-601.

10. Royal College of Obstetricians and Gynaecologists. The investigation and management of the small-for-gestational-age fetus. Green-top Guideline. 2013;31:1-34.

11. Saldanha M, Machado M, Matos C, Pinto F, Barroso R, Carreiro $H$. Recém-nascidos leves para a idade gestacional numa população suburbana. Incidência e factores de risco. Acta Pediatr Port. 2003;1:2532.

12. Drake A, Walker B. The intergenerational effects of fetal programming: non-genomic mechanisms for the inheritance of low birth weight and cardiovascular risk. J Endocrinol. 2004;180:1-16.

13. Sehested L, Pedersen P. Prognosis and risk factors for intrauterine growth retardation. Danish Med J. 2014;61:1-4.

14. Aghamolaei $T$, Eftekhar $H$, Zare S. Risk factors associated with intrauterine growth retardation (IUGR) in Bandar Abbas. J Med Sci. 2007;7:665-9.

15. Conley D, Bennett N. Birth weight and income: interactions across generations. J Health Soc Behav. 2001;42:450-65.

16. McCowan $L$, Horgan R. Risk factors for small for gestational age infants. Best Pract Res Clin Obstet Gynaecol. 2009;23:779-93.

17. Budhathoki S, Poudel P, Bhatta N, Singh R, Shrivastava M, Niraula S, et al. Clinico-epidemiological study of low birth weight newborns in the Eastern part of Nepal. Nepal Med Coll J. 2014;16:190-3.

18. Forbes $\mathrm{K}$, Westwood M. Maternal growth factor regulation of human placental development and fetal growth. J Endocrinol. 2010;207:1-16.

19. Buitendijka S, Zeitlinb J, Cuttinic M, Langhoff-Roosd J, Bottu J. Indicators of fetal and infant health outcomes. Eur J Obstet Gynecol Reprod Biol. 2003;111:66-77

20. Wilcox J. On the importance - and the unimportance - of birthweight. Int J Epidemiol. 2001;30:1233-41.

21. Gill A, Yu V, Bajuk B, Astbury J. Postnatal growth in infants born before 30 weeks' gestation. Arch Dis Child. 1986;61:549-53.

22. Jong F, Monuteaux M, Elburg R, Gillman, Belfort M. Systematic review and meta-analysis of preterm birth and later systolic blood pressure. Hypertension. 2012;59:226-34

23. Barker DJ. Adult consequences of fetal growth restriction. Clin Obstet Gynecol. 2006;49:270-83.

24. Ross M, Beall M. Adult sequelae of intrauterine growth restriction. Semin Perinatol. 2008;32:213-8.

25. Dias C. Vigilância pré-natal e decisão do parto. Nascer Crescer. 2012;21:159-66

26. Fenton T, Kim J. A systematic review and meta-analysis to revise the Fenton growth chart for preterm infants. BMC Pediatr. 2013;13:59.

27. Proença E, Vasconcellos G, Rocha G, Carreira M, Mateus M, Santos I, et al. Displasia broncopulmonar. Consensos em Neonatologia. Lisboa: Sociedade Portuguesa de Neonatologia; 2009.
28. Copel J, Bahtiyar M. A practical approach to fetal growth restriction. Obstet Gynecol. 2014;123:1057-69.

29. Dudley N. A systematic review of the ultrasound estimation of fetal weight. Ultrasound Obstet Gynecol. 2005;25:80-9.

30. Monier I, Ancel P, Ego A, Jarreau P, Lebeaux C, Kaminski M, et al. Fetal and neonatal outcomes of preterm infants born before 32 weeks of gestation according to antenatal vs postnatal assessments of restricted growth. Am J Obstet Gynecol. 2017;216:516.e1-10.

31. Marsál K, Persson P, Larsen T, Lilja H, Selbing A, Sultan B. Intrauterine growth curves based on ultrasonically estimated foetal weights. Acta Paediatr. 1996;85:843-8.

32. Zeitlin J, El Ayoubi M, Jarreau P, Draper E, Blondel B, Kunzel W, et al. Impact of fetal growth restriction on mortality and morbidity in a very preterm birth cohort. J Pediatr. 2010;157:733-9.

33. Monier I, Blondel B, Ego A, Kaminski M, Goffinet F, Zeitlin J. Does the presence of risk factors for fetal growth restriction increase the probability of antenatal detection? A French national study. Paediatr Perinat Epidemiol. 2016;30:46-55.

34. Rodrigues T, Teles T, Miguel C, Pereira A, Barros H. Recém-nascidos leves para a idade gestacional. Influência das curvas padrão de peso ao nascimento no cálculo da sua prevalência e dos factores de risco. Acta Med Port. 1996;9:335-40.

35. Sarquis A, Miyaki M, Cat M. Aplicação do escore CRIB para avaliar o risco de mortalidade neonatal. J Pediatr. 2002;78:225-9.

36. Richardson DK, Corcoran JD, Escobar GJ, Lee SK. SNAP-II and SNAPPE-II: simplified newborn illness severity and mortality risk scores. J Pediatr. 2001;138:92-100.

37. Harsha S, Archana B. SNAPPE-II (Score for Neonatal Acute Physiology with Perinatal Extension-II) in predicting mortality and morbidity in NICU. J Clin Diagn Res. 2015;9:10-2

38. Silveira R, Schlabendorff M, Procianoy R. Valor preditivo dos escores de SNAP e SNAP-PE na mortalidade neonatal. J Pediatr. 2001;77:455-60.

39. Parikh N, Lasky R, Kennedy A, McDavid G, Tyson J. Perinatal factors and regional brain volume abnormalities at term in a cohort of extremely low birth weight infants. PLoS One. 2013;8:1-11.

40. Graça AM, Cardoso K, Costa J, Cowan FM. Cerebral volume at term age: comparison between preterm and term-born infants using cranial ultrasound. Early Hum Dev. 2013;89:643-8.

41. Peterson B, Anderson A, Ehrenkranz R, Staib L, Tageldin M, Colson $\mathrm{E}$, et al. Regional brain volumes and their later neurodevelopmental correlates in term and preterm infants. Pediatrics. 2003;111:939-48.

42. Bruno C, Bengani S, Gomes W, Brewer M, Vega M, Xie X, et al. MRI differences associated with intrauterine growth restriction in preterm infants. Neonatology. 2017;111:317-23.

43. Luque M, Tapia J, Villarroel L, Marshall G, Musante G, Carlo W, et al. A risk prediction model for severe intraventricular hemorrhage in very low birth weight infants and the effect of prophylactic Indomethacin. J Perinatol. 2014;34:43-8.

44. Zeitlin J, Papiernik E, Bréart G, Draper E, Kollée L, MOSAIC Research Group. Presentation of the European project models of organising access to intensive care for very preterm births in Europe (MOSAIC) using European diversity to explore models for the care of very preterm babies. Eur J Obstet Gynecol Reprod Biol. 2005;118:272-4.

45. Schwarzler $P$, Senat MV, Holden $D$, Bernard $P$, Masroor $T$, Ville $Y$ Feasibility of the second-trimester fetal ultrasound examination in an unselected population at 18, 20 or 22 weeks of pregnancy: a randomized trial. Ultrasound Obstet Gynecol. 1999;14:92-7. 